\title{
RESEARCH INTO THE COLLIMATION AND HORIZONTAL AXIS ERRORS INFLUENCE ON THE Z+F LASER SCANNER ACCURACY OF VERTICALITY MEASUREMENT
}

\author{
J. Sawicki, M. Kowalczyk* \\ Department of Photogrammetry, Remote Sensing and Spatial Information Systems, Faculty of Geodesy and Cartography \\ Warsaw University of Technology, Plac Politechniki 1, Warsaw, Poland - sawicki.jm@gmail.com, mikowalczyk@wp.pl,
}

\section{Youth Forum}

KEY WORDS: laser scanning, collimation error, horizontal axis error, engineering geodesy, verticality measurement

\begin{abstract}
:
Aim of this study was to appoint values of collimation and horizontal axis errors of the laser scanner ZF 5006h owned by Department of Geodesy and Cartography, Warsaw University of Technology, and then to determine the effect of those errors on the results of measurements. An experiment has been performed, involving measurement of the test field, founded in the Main Hall of the Main Building of the Warsaw University of Technology, during which values of instrumental errors of interest were determined. Then, an universal computer program that automates the proposed algorithm and capable of applying corrections to measured target coordinates or even entire point clouds from individual stations, has been developed.
\end{abstract}

\section{INTRODUCTION}

\subsection{Motivation}

Laser scanners are often being described as "very fast, reflectorless tacheometers". This is due to a fact, that the same methods of measuring directions and distances are used in both of them. Thus, most of instrument errors related to total stations also apply to laser scanners. Although all such faults are investigated and somehow (either mechanically or electronically) eliminated during professional calibration, it used to be a common practice by surveyors to occasionally inspect them. As far as the process of such "rough calibration" has been widely discussed in context of classical instruments, much fewer propositions apply to laser scanners. The producers recommend to rely on factory calibration and service the device periodically to keep it up to date. Nonetheless, the users should be able to audit their instruments by themselves because the values of instrument errors may change due to mechanical damages, improper storage, variability of measurement conditions and the passage of time. Knowledge of those values is critical to determine survey's accuracy or to apply adequate corrections.

\subsection{Reference to related work}

The laser scanner's calibration is more complex than its total station equivalent, because most often there is no possibility to point the laser beam at specific targets and gather actual distance and direction readings. Some hardware manufacturers might enable those functions by using some dedicated software (Walsh, 2011), but a regular user is limited to acquiring point clouds and as a consequence further analysis has to be based on point data sets.

There has been a set of field procedures for testing terrestrial laser scanners introduced (Reinhard, 2008), but all of those lead the user to checking whether some linear deviation that was acquired exceeds demanded accuracy or not. Although those methods are very fast, neither of them provides instrument errors values, which makes applying proper corrections resulting in elimination of their influence impossible.

More sophisticated investigations of laser scanners accuracy is most often defined as a measure to an independent reference. This sort of research is reasonable for evaluating the accuracy of the whole hardware-software structure, as the precision of 3D laser systems is composed of combination of errors in distance and angle measurements, and in the algorithm for fitting the targets in the point cloud (Kersten, 2009). Other approaches literally describe instrument's observation as "not of real interest" (Staiger, 2005).

Collimation and horizontal axis errors are instrument errors, which cause a misrepresentation of horizontal circle readings. Their influence increases respectively to the vertical angle of sight axis vertical angle. One of the most common type of engineering geodesy survey, where laser scanning technology finds its application, is objects verticality measurement, where high precision is expected. Scanning a tall object, especially from a relatively short distance, leads to very steep sigh axis measurements, which brings the influence of collimation and horizontal axis errors to the extreme.

\subsection{Detailed definitions of investigated errors}

The collimation error exists if the collimation axis ("sight" axis) is not perpendicular to the horizontal axis. The horizontal axis error is present when the horizontal axis is not perpendicular to the vertical axis (Deumlich, 1982).

Collimation error causes the laser beam move within a cone instead of a plane when the prism is revolving. Horizontal axis error effects in tilting the surface within which the collimation axis moves (Figure 1.).

\footnotetext{
*Corresponding author.
} 


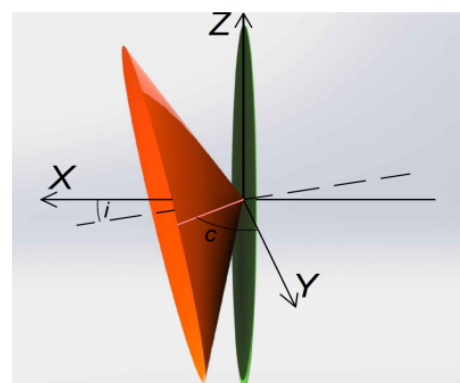

Figure 1. Tilted cone within which the collimation axis moves

The influence of those errors on horizontal circle readings can be calculated with following formulas (Deumlich, 1982):

$$
\begin{gathered}
\varepsilon_{\mathrm{c}}=\frac{\mathrm{c}}{\sin \mathrm{z}} \\
\varepsilon_{\mathrm{i}}=i * \cot (z)
\end{gathered}
$$

where $c$ - collimation error value, $i$ - horizontal axis error value, $z$ - zenith angle

Collimation and horizontal axis errors cause deformations of each line of points obtained. Not only proper corrections to horizontal angles should improve registration accuracy, but also enhance inner geometry of single scans.

\subsection{Aims of the study}

In this study, an algorithm for determining collimation and horizontal axis errors is going to be elaborated. The analysis will be based on test field survey, which could be easily performed by any laser scanner user in the future.

Afterwards, a computer application will be developed, which will fully automate the calculation process based on initially prepared data sets. Additional function of this application will be applying corrections to entire point clouds based on investigated or entered by user instrument errors values.

Collectively, a complete workflow, that could be implemented in actual surveys, for obtaining instrument errors of interest values and eliminating their influence on the measurement, is going to be proposed.

\section{DEVELOPED ALGORITHM}

\subsection{Investigated scanner}

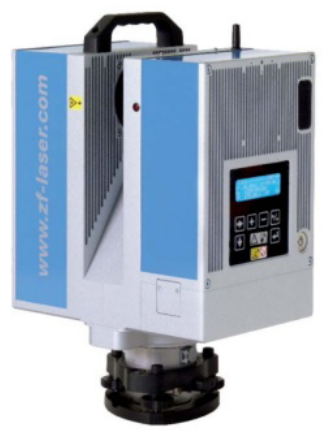

Figure 2. Terrestrial laser scanner ZF 5006h

Terrestrial laser scanner ZF 5006h (Figure 2.) was researched into. Most important feature of this instrument, that affects further analysis, is the way it scans its surrounding. Main part of the device is a rotating prism, which constantly revolves around the horizontal axis of the scanner. The whole device is also slowly rotating around vertical axis relatively to its tribrach. Thus, the prism is scanners equivalent of total stations telescope. During prisms rotation, slant distance is being measured at high frequency for vertical angles of laser beam range $\left(-60^{\circ}, 240^{\circ}\right)$. The whole device needs to rotate by an angle of $180^{\circ}$ to obtain points from the whole 360 scene - half of the scene is observed with face right, the other with face left.

Another important aspect of this piece of hardware is its angle measurement accuracy equal to $77^{\mathrm{cc}}$. Later in this study it could be compared against collimation and horizontal axis errors influence on horizontal circle readings.

During test field measurements, scan resolution was set to High $(3 \mathrm{~mm} / 10 \mathrm{~m})$.

\subsection{Test field and measurements}

Test field (Figure 3.) used in this experiment was founded in the Main Hall of the Main Building of the Warsaw University of Technology. It consisted of numerous paper targets stabilized on walls with with adhesive tape:

- 24 targets located at instruments level

- 24 targets located at 3 floor level

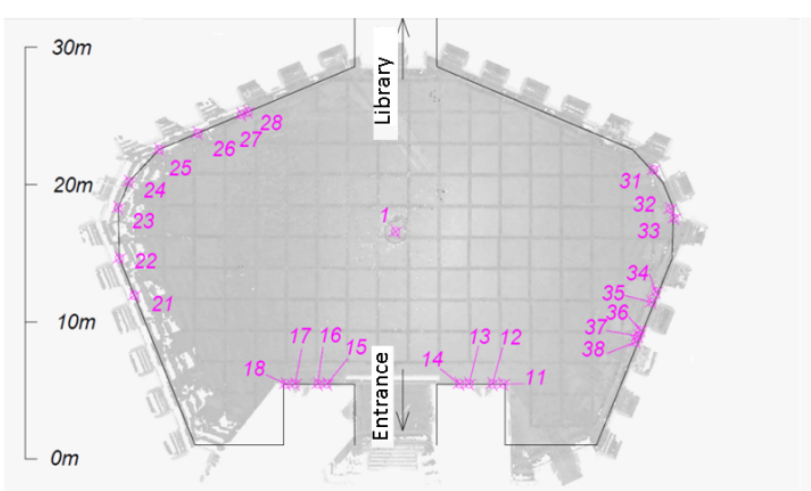

Figure 3. Test field (scanner-level targets only)

Although it was not necessary, in this particular implementation each target at instruments level had its corresponding one at 3 floor level.

To designate collimation and horizontal axis errors values, particular targets need to be observed at least twice - with both faces of the instrument. In order to allow this, the instrument will be removed from its tribrach, and reinstalled after being rotated by an angle of approximately $120^{\circ}$. The scanner can be set on the tribrach in 3 different positions - thus, three full scene scans will be obtained from the same station.

It is crucial to maintain station coordinates. Correlatively to average distance to targets, horizontal displacement of the station affects the accuracy of appointed values. Minimal vertical station's shift, caused for example by the construction of a tribrach can be neglected.

\subsection{Principles of proposed algorithm}

Coordinates of targets in local coordinate system (Figure 4.), whose origin is located in the center of scanners prism, could be easily calculated back to angle and distance measurements accordingly to following formulas: 


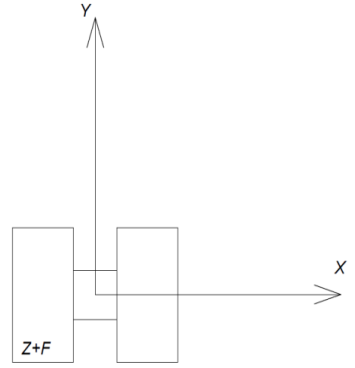

Figure 4. Local scanners coordinate system (seen from top)

(face right) $\begin{aligned} & s=\sqrt{x^{2}+y^{2}+z^{2}} \\ & Z=100^{g}-\arcsin \left(\frac{z}{s}\right) \\ & \text { (face left) } \quad Z=300^{g}+\arcsin \left(\frac{z}{s}\right)\end{aligned}$
(1st and 3rd quadrant) $H z=\tan ^{-1}\left(\frac{x}{y}\right)$
(2nd and 4th quadrant) $H z=200^{g}-\tan ^{-1}\left(\frac{-x}{y}\right)$

where $x, y, z$ - point coordinates in local coordinate system, $s$ - slant distance, $Z$ - zenith angle, $H z$ - horizontal angle

The horizontal circle readings need to be reduced to mutual coordinate system. It is possible to obtain precise values of angles the scanner was rotated in its tribrach, by comparing observations to corresponding targets acquired with the same face of the instrument, as their substraction is free of collimation and horizontal axis errors influence.

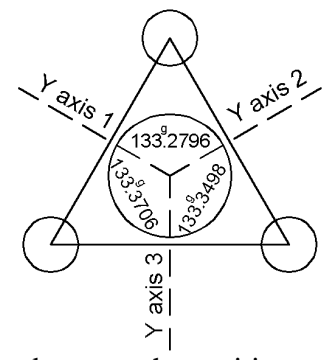

Figure 5. The angles between the positions of the scanner in the tribrach

Although use of all targets is possible, it is recommended to perform above calculation only on observations to scanner-level targets. This is not only due to better point cloud resolution and scanning angle, but these readings are free of deviations due to minimal out of level drift. After these angles are designated, all horizontal angle readings can be reduced to first positions of the scanner coordinate system. Distance and reduced angle measurements to particular targets can now be confronted to appoint any blunders, especially the ones caused by station or target displacement.

Following such verification, corresponding observations obtained with the same face of the instrument are now to be averaged. Finally, each target has its face left and face right observations evaluated. This stage being completed, further calculations leading to evaluation of collimation and horizontal axis errors may process identically as for total stations. The value of collimation error can be assessed by the following formula when face left and face right pair of horizontal circle readings to distinct target is given:

$$
c=\frac{H z_{F R}-H z_{F L} \pm 200^{g}}{2}
$$

where $c$ - collimation error value, $H z_{F R}$ - face right horizontal angle reading, $H z_{F L}$ - face left horizontal angle reading

Adequately, horizontal axis error can be calculated:

$$
i=\frac{H z_{F R}-H z_{F L} \pm 200^{g}}{2} \tan (Z)-\frac{c}{\cos (Z)}
$$

where $i$ - horizontal axis error value, $c$ - collimation error value, $H z_{F R}$ - face right horizontal angle reading, $H z_{F L}$ - face left horizontal angle reading, $Z$ - zenith angle

Depending on the number of targets set up in the test field, numerous evaluations can be executed. Average values of instrument errors of interest values are considered final results. Additionally, standard deviations of designations can be calculated, although they are not comparable to angle measurement accuracy due to variability of point cloud density.

\subsection{Results of the research}

For additional control, two independent observation data sets obtained with the test scanner were processed in accordance with above algorithm. The results were as follows:

- For 1st observations set:

$$
\begin{array}{ll}
\circ & c=-461^{c c}+/-37^{c c} \\
\circ & i=169^{c c}+/-207^{c c}
\end{array}
$$

- For 2nt observations set:

$$
\begin{array}{ll}
\circ & c=-454^{c c}+/-21^{c c} \\
\circ & i=246^{c c}+/-100^{c c}
\end{array}
$$

Average values of those evaluations were considered final:

$$
\begin{array}{ll}
\circ & c=-457^{c c} \\
\circ & i=-208^{c c}
\end{array}
$$

To illustrate the linear influence of this angle values, following conditions of possible survey were assumed:

- objects height $50 \mathrm{~m}$

- distance between the scanner and object $30 \mathrm{~m}$

Influence of appointed instrument errors values was calculated with formulas (1) and (2) and presented on Figure 6. Both collimation and horizontal axis introduce significant deviations.

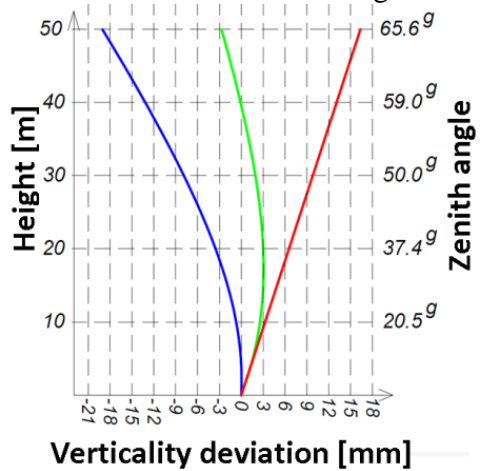

Figure 6. Verticality deviation due to researched errors (blue collimation error influence, red - horizontal axis error influence, green - final deviation caused by both errors) 
In this specific scanner they have different signs and mutually reduce their impact on measurements to approximately $3 \mathrm{~mm}$ within the devices range. Worth noticing, that opposite sign of any of the values would result in errors of verticality measurement reaching almost $5 \mathrm{~cm}$.

\subsection{Observation corrections}

Once the values of instrument errors are evaluated, their influence (accordingly with formulas (1) and (2)) on observations can be. Applying such corrections, based on final values of collimation and horizontal axis errors, to both observations sets obtained during test field measurement resulted with following residual errors:

- For 1st observations set:

$$
\begin{array}{ll}
\circ & c=-2^{c c}+/-36^{c c} \\
\circ & i=-41^{c c}+/-211^{c c}
\end{array}
$$

- For 2nd observations set:

$$
\begin{array}{ll}
\circ & c=4^{c c}+/-20^{c c} \\
\circ & i=37^{c c}+/-97^{c c}
\end{array}
$$

Elimination of even minimal systematic should result in improved registration, better single point clouds geometry, lower noise on surfaces in registered point clouds etc.. For additional, independent control, actual survey scans were obtained from a project consisting of 3 scanner stations and 5 targets, in which tested scanner was used. The stations were registered to mutual coordinate system - after applying suggested corrections to single point clouds RMS error of the registration improved from $11 \mathrm{~mm}$ to $8 \mathrm{~mm}$.

\section{COMPUTER APPLICATION}

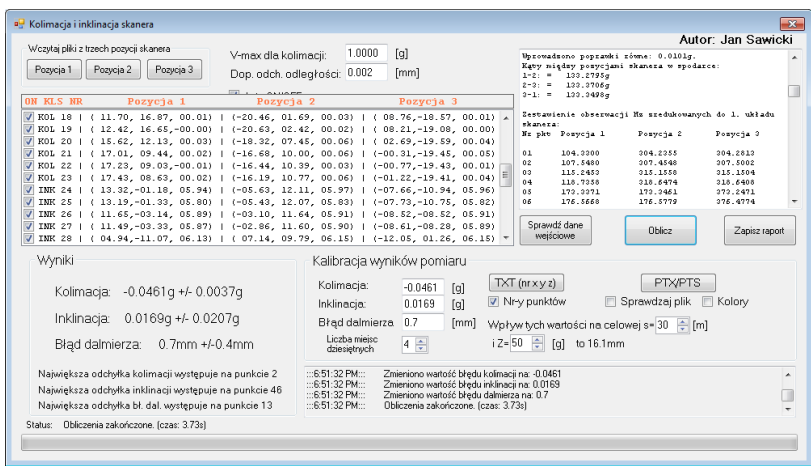

Figure 7. Computer application for proposed algorithm implementation

All above calculation, can be easily accomplished with authorial computer program. After test field measurements, coordinates of all targets need to be evaluated in local coordinate systems. Text files containing such point data sets should be loaded into the program, rest of the calculation is carried out automatically.

Based on either default or input by user parameters the program decides which targets should be used for collimation, and which for horizontal axis error evaluation. Any blunders due to target displacement are appointed and excluded from calculation. Depending on number of targets available, user can exclude further observations to decrease standard deviations of evaluated values. Additionally to instrument errors of this research's interest, the program designates any constant offset in slant distance measurement (negligible value of $0.6 \mathrm{~mm}$ for this particular device).
To let the user know, how much computed angular values influence linear accuracy, a simple calculator has been implemented - to determine the linear error it expects an input of zenith angle and length of the sight axis.

Last but not least, above application is able to apply corrections to entire point clouds due to investigated instrument error values - input acceptable are *.PTS and *.PTX files.

\section{PROPOSED WORKFLOW}

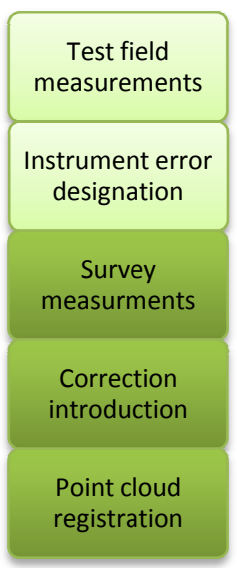

Figure 8. Proposed workflow

Test field measurements can be performed periodically and designated values may serve plenty laser scanning projects. In specific scenarios, inaccuracy of instrument's calibration can be noticed much after actual survey measurements, which normally would enforce numerous scans to be retaken. Assuming stability of instrument errors values, they can be acquired after field measurements. Thus, inappropriately calibrated point data sets can be recovered and extra fieldwork eliminated.

Although it is possible to introduce corrections to registered point clouds, it is recommended to apply them before the registration process as not only it improves inner geometry of single point clouds, but also increases registration accuracy.

\section{CONCLUSIONS}

Both investigated instrument errors introduce significant linear deviations to verticality measurement. Not only each half of the scan (obtained either with face left or face two) is deformed, but the halves are inappropriately united to mutual coordinate system, which results in extra registration errors and noticeable thresholds along the joint.

Thus, keeping the instrument's calibration up to date is crucial for obtaining accurate measurements. Surveyors are not limited to relying on manufacturers calibration, but various of methods are being developed for terrestrial laser scanners inspections.

Ability to apply observation corrections distinguishes proposed algorithm from other similar approaches. In future, other specific instrument errors could be researched into, which might result in enabling laser scanner users to fully examine their devices. 


\section{REFERENCES AND SELECTED BIBLIOGRAPHY}

Deumlich, F., 1982. Surveying Instruments, Walter de Greuter, New York

Walsh, G., 2011. Leica ScanStation: Calibration and QA, Leica Geosystems, San Ramon.

Reinhard, G., 2008. Field Procedures for Testing Terrestrial Laser Scanners (TLS), A Contribution to a Future ISO Standard, Stockholm, FIG Working Week.

Kersten, T., Mechelke, K., Lindstaedt, M., Sternberg H, 2009, Methods for Geometric Accuracy Investigations of Terrestrial Laser Scanning Systems, Photogrammetrie Fernerkundung Geoinformation, Heft 4, Stuttgart, pp. 301-314.

Staiger, R., 2005. The Geometrical Quality of Terrestrial Laser Scanners (TLS), Cairo, FIG Working Week. 